\section{Valor nutritivo do bagaço de cana-de-açúcar tratado por soluções alcalinas através de digestibilidade aparente com ovinos}

\author{
Evaluation of nutritive value of sugar cane bagasse treated with \\ alkaline solutions through digestibility trial with sheep
}

Sylvia SONKSEN; Carlos de Sousa LUCCI²; Laércio MELOTTI²

\author{
CORRESPONDENCE TO: \\ Carlos de Sousa Lucci \\ Departamento de Nutrição e \\ Produção Animal \\ Faculdade de Medicina \\ Veterinária e Zootecnia da USP \\ Caixa Postal 23 \\ Caixa Postal 23 caxias Norte, 225 \\ Av. Duque de Caxias Norte, 225 \\ $13630-970$ - Pirassununga - SP - \\ Brasil \\ e-mail: cslucci@usp.br \\ 1 - Cati - SAASP \\ 2 - Departamento de Nutrição e \\ Produção Animal \\ Faculdade de Medicina \\ Veterinária e Zootecnia da USP
}

\title{
RESUMO
}

Bagaço de cana-de-açúcar (BCA) foi tratado por imersão em água (B), ou em solução de soda a $2 \%$ (D), ou em solução de cinzas de madeira a $30 \%$ (C), durante 6 horas, em sacos de tela de náilon que, em seguida, foram erguidos para escorrer o excesso de líquido, o qual era reaproveitado ou ainda suplementado por concentrado contendo tamponantes (A), com o intuito de estudar seu valor nutritivo através de ensaio de digestibilidade in vivo. Foram utilizados 12 ovinos, machos, castrados, que foram arreados para colheita total de fezes e permaneceram em gaiolas metabólicas. O delineamento estatístico foi o de blocos ao acaso, com 3 blocos de animais. A digestibilidade aparente da matéria seca (MS) e da matéria orgânica (MO) foi significativamente menor, e a da fibra bruta foi maior para o tratamento $D(p<0,05)$. O tratamento $D$ foi eficiente para melhorar digestibilidade aparente da fibra bruta (FB) da ração.

UNITERMOS: Digestibilidade; Ovinos; Bagaço de cana-de-açúcar.

\section{INTRODUÇÃO E REVISÃO DE LITERATURA}

$\mathrm{O}$ bagaço de cana-de-açúcar (BCA), subproduto da agroindústria açucareira, tem sua digestibilidade associada à quantidade de lignina presente, a qual interfere no maior ou menor aproveitamento da celulose e hemicelulose (Jung; Fahey Jr. ${ }^{12}$, 1983, e Jung "1, 1989). O subproduto contém $42 \%$ de celulose, 29,4\% de hemicelulose e 20,2\% de lignina (Paturau ${ }^{22}, 1981$ ), ou de $10 \%$ a $20 \%$ de lignina (Boin et al. $^{3}, 1987$ ).

A composição química do BCA varia de $1,1 \%$ a $3,3 \%$ de PB, $2,0 \%$ a $5,9 \%$ de MM, $84,0 \%$ a $94,6 \%$ para FDN e $58,0 \%$ a $68,0 \%$ para FDA (Ibrahin; Pearce, 1983 ; Kumar et al. ${ }^{14}$, 1982; Lamas et al. ${ }^{15}$, 1979; Molina et al. $.^{17}, 1983$ ). O NRC ${ }^{21}$ (1988) também mostra $46,6 \%$ de MS, $1,5 \%$ de PB, $49,0 \%$ de FB e $5,5 \%$ de cinzas. Nicholson (1984) apud Morgullis ${ }^{18}$ relatou teores de 26,0\% a 46,0\% de NDT para o BCA.

Klopfenstein ${ }^{13}$ (1978) mostra que, com o tratamento por álcalis, há quebra das ligações entre a lignina e a celulose ou hemicelulose, sem remoção da lignina, visto que seu conteúdo não é reduzido com o tratamento. No entanto, para Feist et al. ${ }^{6}(1970)$, alguma sílica e lignina podem ser dissolvidas e as ligações intermoleculares entre grupos do ácido urônico da hemicelulose e grupos de hidroxila da celulose podem ser hidrolizadas pelo $\mathrm{NaOH}$, tornando-o um produto mais digestível. Segundo Jackson ${ }^{9}$ (1977), as alterações que ocorrem na composição e organização da parede celular com o tratamento alcalino seriam solubilização parcial da hemicelulose, lignina e sílica na hidrólise das ligações ésteres entre a hemicelulose e o ácido urônico; também há redução da resistência das pontes de hidrogênio entre moléculas de celulose, provocando a expansão das mesmas.

Existem trabalhos sobre os efeitos da aplicação de $\mathrm{NaOH}$ e
KOH em matérias fibrosas, como o de Carmona; Greenhalgh (1972), que alimentaram ovinos com palha de cevada tratada pelo processo de imersão em soluções de soda com concentrações de $0,5 \%$ a $4,0 \%$. Em relação à digestibilidade. observaram que houve aumento linear até a concentração de $2 \%$ na solução de $\mathrm{NaOH}$, que depois permaneceu constante. Jayasuriya; Owen ${ }^{10}$ (1975) realizaram experimento para determinar a quantidade ótima de álcali e, dentre as porcentagens utilizadas, observaram que houve aumento na digestibilidade após os tratamentos com soluções de $2 \%$ e $4 \%$, principalmente para a fibra bruta (FB). O consumo de MS foi significativamente maior para os tratamentos com soluções a $2 \%$. Rexen; Thomsen ${ }^{24}$ (1976) estudaram um método industrial em que as paIhas eram tratadas por aspersão com uma solução de $30 \%$ de $\mathrm{NaOH}$ e a seguir adicionadas de melaço e peletizadas. Na composição química observaram que as porcentagens de hemicelulose diminuíram, enquanto as de celulose permaneceram constantes. Encontraram também aumento linear na digestibilidade in vitro da matéria orgânica (MO), com concentrações crescentes de álcali. Entretanto, dosagens acima de $4 \%$ a $5 \%$ de $\mathrm{NaOH}$ não melhoraram a digestibilidade in vivo, possivelmente em virtude do aumento no consumo de sódio e de água, acarretando velocidade maior de passagem da ingesta pelo rúmen. Molina et al. ${ }^{17}$ (1983) pulverizaram o BCA com $\mathrm{NaOH}$, nas proporções de $2 \%, 4 \%$ e $6 \%$ da MS, encontrando diminuição linear na fração FDN; o BCA não tratado apresentou $93,6 \%$ de FDN e o tratado com $6 \%$ de $\mathrm{NaOH}, 72,7 \%$. Houve aumento do teor de cinzas do BCA com emprego de maiores níveis de $\mathrm{NaOH}$. Cabello et $\mathrm{al}^{4}{ }^{4}$ (1981) trataram o BCA com soda, em níveis de $4 \%$ a $12 \%$ na MS. No nível de $8 \%$ de $\mathrm{NaOH}$, a digestibilidade in vitro foi igual a $80 \%$, enquanto para o BCA não tratado foi $20 \%$. Ibrahin; Pearce ${ }^{\mathrm{y}}$ (1983) observaram que os coeficientes de digestibilidade da MS in vitro do BCA sem tratamento e 
SONKSEN. S.; LUCCI, C.S.; MELOTTI, L.. Valor nutritivo do bagaço de cana-de-açúcar tratado por soluções alcalinas através de digestibilidade aparente com ovinos Braz. J. vet. Res. anim. Sci., São Paulo, v. 34, n. 5, p. 296-299, 1997

o tratado por soda a $9 \%$ foram $32,8 \%$ e $57,1 \%$, respectivamente. Nolte et al. ${ }^{19}$ (1987) utilizaram soluções alcalinas com cinzas de madeira de $10 \%$ a $50 \%$ para tratamento da palha de trigo por imersão. Os valores encontrados na digestibilidade in vitro da MS aumentaram significativamente para todos os tratamentos, e para MO foram $33,4 \% ; 41,2 \% ; 38,5 \%$ e $36,6 \%$ para palha não tratada, tratada com $\mathrm{NaOH}$ a $1 \%$, com solução de cinzas a $30 \%$ e a $15 \%$, respectivamente. Adebowale' ( 1985 ) realizou ensaio de digestibilidade com caprinos em que a palha de milho foi tratada por solução de soda e cinzas. O tratamento por $\mathrm{NaOH}$ apresentou melhores valores da digestibilidade da MS, concentração a $1 \%$ de $\mathrm{NaOH}$, e para a MO, com $2 \%$ de $\mathrm{NaOH}$. Ramirez et al. ${ }^{23}$ (1991) realizaram ensaio de digestibilidade com ovinos para avaliar os efeitos do tratamento alcalino (cinzas e soda) sobre a palha de sorgo e encontraram para a digestibilidade da MS e da FDN, $53,0 \%$ e $51,5 \%$ para $0 \%$ de cinzas e $60.0 \%$ e $63.1 \%$ para $20 \%$ de cinzas, respectivamente. Morgullis $^{18}$ (1992) tratou o BCA. que constituía $60 \%$ das dietas, com soluções de $\mathrm{NaOH}$ a $2 \%$ (A), cinzas de madeira $30 \%$ (B) ou água (C) e concluiu que os tratamentos alcalinos melhoraram a digestibilidade aparente com carneiros cujos coeficientes de digestibilidade para MS, MO e FDN foram: $59,59 \% ; 59,29 \%$ e $59.22 \%$, para o tratamento A; $43.18 \% ; 48.18 \%$ e $37.78 \%$, para B; e $35,34 \%, 37,51 \%$ e $25,88 \%$ para o $\mathrm{C}$, respectivamente.

O objetivo deste experimento foi estudar o efeito da hidrólise alcalina sobre o valor nutritivo do BCA em rações para ovinos, contendo $30 \%$ de $\mathrm{BCA}$ e $70 \%$ de concentrado, através da digestibilidade aparente.

\section{MATERIAL E MÉTODO}

Foram utilizados 12 animais, ovinos machos, castrados, da raça Ideal, com aproximadamente 1 ano de idade. O trabalho foi realizado na Faculdade de Medicina Veterinária e Zootecnia da Universidade de São Paulo, no Campus Administrativo de Pirassununga (SP).

Quatro tratamentos foram estudados sendo: 1) Trat. A - BCA tratado com água adicionada de tampões (bicarbonato de sódio $1,1 \%$ e óxido de magnésio $0.7 \%$ ); 2) Trat. B - BCA tratado com água (testemunha); 3) - Trat. C - BCA tratado com solução a 30\% de cinzas de madeira; 4) Trat. D - BCA tratado com solução a $2 \%$ de hidróxido de sódio $(\mathrm{NaOH})$. O BCA (aproximadamente $100 \mathrm{~kg}$ ) era colocado em sacos de tela de náilon e com auxílio de uma catraca era submerso em caixas para água com capacidade de $1000 \mathrm{I}$, sendo uma para cada tratamento. Cada saco do BCA ficava durante 6 horas em imersão em 700 I de solução, quando então era suspenso por 18 horas sobre as respectivas caixas, visando escorrer o excesso de líquido, bem como reaproveitá-lo. O BCA era fornecido juntamente com concentrados nos quatro tratamentos constituídos de $30 \%$ de $\mathrm{BCA}$ e $70 \%$ de concentrados em base da matéria seca (milho moído + farelo de algodão + premix ).

Na Tab. I estão os ingredientes utilizados nas rações dos quatro tratamentos.

As rações experimentais foram formuladas de acordo com as exigências nutricionais recomendadas pelo $\mathrm{NRC}^{20}$ (1985) para ovinos.

O ensaio compreendeu colheita tolal de fezes, utilizando-se arreios de digestibilidade e gaiolas metabólicas.

Tabela 1

Ingredientes usados nas rações dos diversos tratamentos na MS, em porcentagens. (Pirassununga, São Paulo, 1990).

\begin{tabular}{lrrrr}
\hline $\begin{array}{l}\text { TRATAMENTO/ } \\
\text { INGREDIENTE }\end{array}$ & $\mathrm{A}$ & $\mathrm{B}$ & $\mathrm{C}$ & $\mathrm{D}$ \\
\hline Bagaço & 30,0 & 30,0 & 30,0 & 30,0 \\
Grão de milho moído & 52,06 & 54,54 & 54,54 & 54,54 \\
Farelo de algodão & 12,11 & 11,41 & 11,41 & 11,41 \\
Cloreto de potássio & 0,36 & 0,36 & 0,36 & 0,36 \\
Premix mineral & 2,67 & 2,67 & 2,67 & 2,67 \\
Premix vitamínico & 1,02 & 1,02 & 1,02 & 1,02 \\
Bicarbonato de sódio & 1,07 & - & - & - \\
Oxido de magnésio & 0,71 & - & - & - \\
\hline
\end{tabular}

Foi desenvolvido em três fases: sendo a primeira pré-experimental, com 10 dias de duração; a segunda com 9 dias, para adaptação às gaiolas; e a terceira com 5 dias, conforme método descrito em Melotti; Lucci't (1969).

Tabela 2

Resultados das análises bromatológicas dos ingredientes das rações completas com base na matéria seca, em porcentagens. (Pirassununga, São Paulo, 1990).

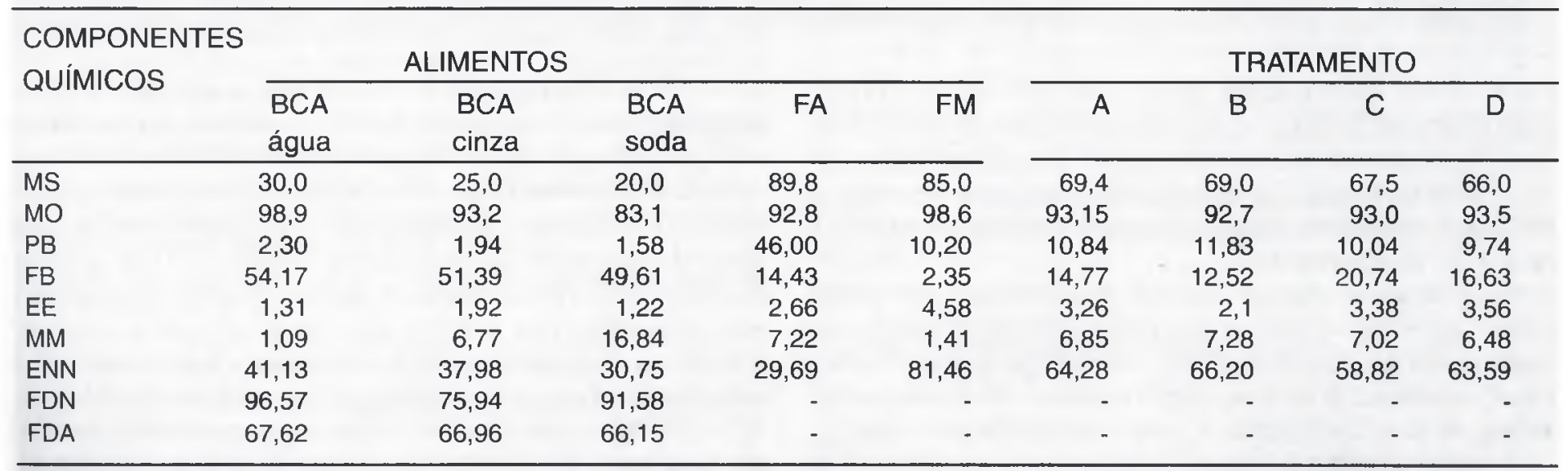

FA = farelo de alondãn $F M=$ milhn (fı ı́á) 
SONKSEN, S.: LUCCI. C.S.: MELOTTI, L.. Valor nutritivo do bagaço de cana-de-açúcar tratado por soluçöes alcalinas através de digestibilidade aparente com ovinos

Braz. J. vet. Res. anim. Sei., São Paulo, v. 34. n. 5, p. 296-299, 1997

Os alimentos eram fornecidos em duas refeições, às 8 horas e às 15 horas, e o BCA misturado ao concentrado dentro do cocho. Água era fornecida três vezes ao dia, em baldes.

No período experimental, as fezes de cada animal eram colhidas diariamente, pesadas e retiradas alíquotas de $10 \%$ que eram secas em estufa a $65^{\circ} \mathrm{C}$, para determinação da MS. O mesmo procedimento era feito para o total de urina do qual também era retirada uma amostra de $10 \%$, acondicionada em vidro e conservada em refrigerador para determinação do nitrogênio. Dos alimentos fornecidos eram tomadas amostras diárias, em torno de $100 \mathrm{~g}$ e secas a $65^{\circ} \mathrm{C}$.

Após a secagem, tanto as fezes como o alimento eram moídos para encaminhamento ao laboratório para análise bromatológica, de acordo com a $\operatorname{AOAC}^{2}(1965)$.

O delineamento experimental escolhido foi o de blocos casualizados (Gomes ${ }^{7}, 1985$ ).

\section{RESULTADOS E DISCUSSÃO}

Na Tab. 2 são encontradas as análises bromatológicas dos ingredientes e das rações completas, em porcentagem na MS.

O BCA utilizado no presente trabalho apresentou dados dentro dos padrões já descritos por Ibrahin; Pearce ${ }^{8}$ (1983); Kumar et al. ${ }^{14}$ (1982); Lamas et al..$^{15}$ (1979); Molina et al. ${ }^{17}$ (1983) e o NRC ${ }^{21}$ (1988).

O teor de matéria mineral (MM) presente no BCA tratado por soluções alcalinas foi alto, principalmente no tratado por soda a $2 \%$.

Na Tab. 3 estão os resultados da ingestão de MS, consumo de água. excreção de urina, os coeficientes de digestibilidade (CD) e os coeficientes de variação.

Nas ingestões de MS ou de água não foram detectadas dife- foi maior para o tratamento com soda. Apesar de a ingestão de MS e o consumo de água não terem sido maiores para soda, os valores numéricos foram maiores para esses parâmetros e a excreção de urina foi significativamente aumentada no tratamento por soda. Segundo Nolte et al. ${ }^{19}$ ( 1987), a quantidade de urina excretada está diretamente relacionada com a ingestão de água.

$\mathrm{O}$ tratamento do $\mathrm{BCA}$ por $\mathrm{NaOH}$ (D) resultou em teores de minerais elevados, da ordem de $16 \%$. A ingestão de água mostrou resultados estatisticamente semelhantes, talvez pela magnitude dos coeficientes de variação. A maior ingestão de água provavelmente esteve ligada ao excesso de sódio circulante que é eliminado pelos rins (Rexen; Thomsen ${ }^{24}$, 1976, e Teh of al. ${ }^{25}$ 1987).

Para o tratamento D, a excreção de urina ocorreu em quantidade superior à encontrada no tratamento testemunha (B). Rexen; Thomsen $^{24}$ (1976) afirmaram que o maior consumo de água, para eliminar íons sódio pela urina. implica maior velocidade de passagem da ingesta pelo rúmen e diminuição na sua digestibilidade, o que pode ter ocorrido no caso de MS, MO e ENN do BCA tratado por soda, no presente experimento. Esses resultados conflitam com os de Nolte et al. ${ }^{19}$ (1987) que mostraram melhor digestão de MO pelo tratamento de palhas por soda, em relação às palhas não tratadas, e com os de Ramirez et al..$^{23}$ (1991), que obtiveram melhor digestão de MS e MO de palhas com tratamentos por cinzas de madeira a $20 \%$ (em relação ao da testemunha).

Contudo a digestão da fibra foi mais alta $(45,0 \%)$, embora não significativamente mais alta, para o tratamento $\mathrm{D}$ (soda a $2 \%$ ), em relação ao da testemunha $(\mathrm{B})$; e mais baixa para o tratamento $\mathrm{A}$ (com tampões), em relação ao tratamento D.

Já a digestibilidade da fibra bruta foi maior para o tratamento com soda $(45,07 \%)$ em relação à água mais tampões $(32,62 \%)$, concordando com Morgullis ${ }^{18}$ (1992), e a digestibilidade dos

Tabela 3

Ingestão de MS, consumo de água, excreção urinária e dados de digestibilidade. (Pirassununga - 1990).

\begin{tabular}{lccccccccc}
\hline TRATAMENTO & \multicolumn{3}{c}{ CONSUMO } & \multicolumn{3}{c}{ EXCR. URINA } & \multicolumn{3}{c}{ CD\% } \\
\hline & MS(\% PV) & MS(g/d) & Água(l/dia) & ml/dia & MS & MO & FB & PB & ENN \\
\hline A & 3,7 & 786 & 1,326 & $516 \mathrm{ab}$ & $65,50 \mathrm{ab}$ & $65,86 \mathrm{ab}$ & $13,77 \mathrm{~b}$ & 62,83 & $74,60 \mathrm{a}$ \\
$\mathrm{B}$ & 3,1 & 578 & 1,076 & $323 \mathrm{~b}$ & $69,89 \mathrm{a}$ & $69,75 \mathrm{a}$ & $32,62 \mathrm{ab}$ & 63,32 & $78,03 \mathrm{a}$ \\
$\mathrm{C}$ & 2,9 & 626 & 1,116 & $451 \mathrm{ab}$ & $63,36 \mathrm{~b}$ & $62,88 \mathrm{ab}$ & $28,39 \mathrm{ab}$ & 54,20 & $71,71 \mathrm{ab}$ \\
$\mathrm{D}$ & 3,4 & 706 & 1,593 & $973 \mathrm{a}$ & $61,16 \mathrm{~b}$ & $60,72 \mathrm{~b}$ & $45,07 \mathrm{a}$ & 55,90 & $65,79 \mathrm{~b}$ \\
CV\% & 12,9 & 20,9 & 37,1 & 3,9 & 4,1 & 20,1 & 6,4 & 3,3 & \\
\hline
\end{tabular}

Letras diversas na mesma coluna acusam dados significativamente diferentes $(p<0,05)$.

renças estatisticamente significativas. A excreção de urina foi maior no tratamento BCA por soda (D) do que no tratamento BCA por água (B); no tratamento por cinzas o valor foi intermediário.

Contrariamente, Adebowale' (1985) encontrou ingestões de MS significativamente maiores para palhas tratadas com $\mathrm{NaOH}$, em relação às não tratadas.

As digestibilidades de MS e MO foram menores nos bagaços tratados por soda (D) que nos imersos em água (B), resultados que contrariam os de Morgullis ${ }^{18}$ (1992) e Ramirez ef al. ${ }^{23}$ (1991). Já a digestibilidade da FB foi maior para o tratamento com soda, concordando com Morgullis ${ }^{18}$ (1992), e menor para o ENN para soda.

Não ocorreram diferenças estatisticamente significativas na ingestão da MS e consumo de água, enquanto a excreção de urina extrativos não nitrogenados foi menor para a soda $(65.79 \%) \mathrm{em}$ comparação com o controle $(78,03 \%)$. A análise dos resultados deve ponderar que a menor digestibilidade do ENN na dieta com $30 \%$ de BCA tratado por soda (D) provavelmente não é compensada pelo aumento da digestão da fibra, já que MS e MO das dietas do tratamento D foram menos digeridas. O teor de FDN foi menor para o tratamento com soda em relação ao da testemunha; enquanto o teor de FDA apresentou valor mais próximo. isto provavelmente pela ação da soda sobre a hemicelulose. Por outro lado, existe a possibilidade de que as digestibilidades de MS e MO foram menores no tratamento por soda (D), em relação ao controle $(B)$, graças à maior taxa de passagem da ingesta pelo aparelho digestivo no tratamento D. O emprego de tam- 
pões (tratamento A) diminuiu, embora não significativamente, os valores da digestibilidade de MS, MO e ENN, em relação ao controle, talvez pelo mesmo motivo.

\section{CONCLUSÕES}

O presente experimento permitiu elaborar as seguintes conclusões:
1) A digestibilidade aparente da fibra bruta foi maior na di eta contendo $\mathrm{BCA}$ tratado por $\mathrm{NaOH}$ a $2 \%$, em relação ao tratamento água + tampões;

2) A digestibilidade de MS, MO e ENN foi inferior nas rações com BCA tratado por soda, em relação ao tratamento controle;

3) O emprego de tratamento por soda melhorou a digestibilidade da fibra da ração. No entanto, a digestão de MS, MO e ENN foi menor

\section{SUMMARY}

Sugar cane bagasse (SCB) was immersed in water (B), or in sodium hydroxide solution $2 \%$ (D), or in wood ashes solution $30 \%(C)$, or it was suplemented with concentrate mixture containing buffers (A) to evaluate its nutritive value through an in vivo digestion trial. Twelve, male, castrated sheep were allocated in metabolic cages with sampling of total fecal output. Animals were assigned in a randomized block design. Dry matter and organic matter digestibilities of treatments were lower, and crude fiber showed higher values $(p<0.05)$. As a conclusion, treatment $D$ was efficient to improve apparent crude fiber digestibility of the ration.

UNITERMS: Digestibility; Sheeps; Sugar cane bagasse.

\section{REFERÊNCIAS BIBLIOGRÁFICAS}

1 - ADEBOWALE, E.A. Organic waste ash as possible source of alkali for animal feed treatment. Animal Feed Science and Technology, v.13, n. 314, p.237-48, 1985

2- AOAC. ASSOCIATION OF OFFICIAL AGRICULTURAL CHEMISTS. Official and methods of analysis. 10. ed. Washington. A.O.A.C., 1965.957 p.

3 - BOIN, C.; MATTOS, W.R.S.; D'ARCE, R.D. Cana-de-açúcar e seus subprodutos na alimentação de ruminantes. In: PARANHOS, S.B. Cana-de-açúcar cultivo e utilização. Campinas, Fundação Cargill. 1987. p.805-50.

4 - CABELLO, A.; CONDE, J.; OTERO, M.A. Prediction of the degradability of sugarcane cellulosic residues by indirect methods. Biotechnology and Bioengineering. v.23, p.2737-45, 1981.

5-CARMONA, F.; GREENHALGH, J.F.D. The digestibility and acceptability to sheep of chopped or milled barley straw soaked or sprayed with alkali. Journal of Agricultural Science, v.78, n. 3, p.477-8.5, 1972

6 - FEIST. W.C.; BAKER, A.J.; TARKOW, H. Alkali requeriments for improving digestibility of hardwoods by rumen microorganisms. Journal of Animal Science, v.30, n.5, p.832-5, 1970 .

7-GOMES, F. P. Curso de estatística experimental. Piracicaba, FEALQ-USP, 1985 $384 \mathrm{p}$

8 - IBRAHIN, M.N.M.; PEARCE, G.R. Effects of chemical pretreatment on the composition and in vitro digest ibility of crop by-products. Agricultural Wastes. v.5, n. 3, p.135-56, 1983

9 - JACKSON, M.G. Review article: the alkali treatment of straws. Animal Feed Science and Technology, v.2, n. 2, p. 105-30, 1977

10- JAYASURIYA, M.C.N.; OWEN, E. Sodium hydroxide treatment of barley straw: effect of volume and concentration of solution on digestibility and intake by sheep. Animal Production. v.21, n. 3, p. 313-22, 1975.

11 - JUNG, H.G. Forage lignins and their effects on liber digestibility. Agronomy Journal, v. 81, n. 1, p. 33-8, 1989.

12 - JUNG, H.G.; FAHEY JR.. G.C. Interaction among phenolic monomers and in vitro fermentation. Journal of Dairy Science, v. 66, n.6, p. 1255-63, 1983.

13 - KLOPFENSTEIN, T.J. Chemical treatment of crop residues. Journal of Animal Science, $v .46, n .3$, p.84I-8, 1978

14 - KUMAR, A.; VERMA, D.N.; DASS, R.S.; SINGH, U.B. Effect of concentration and period of amonia treatment on the chemical composition and nutritive value of sugar cane bagasse. Indian Journal of Nutrition and Dietetics, v. 19, p.381-

\section{$8,1982$.}

15 - LAMAS, G.L.; SHIMADA, A.S.; RUEL.A, S.C.; ZUNGA, H.M. Estudio del valor alimentício de subproductos de la cana de azucar com bovinos en corral Tecnica Pecuaria en México, v.36. p. 59-64, 1979

16 - MELOTTI, L.; LUCCI, C.S. Determinação do valor nutritivo de capins napier e feno através de ensaio de digestibilidade (aparente) com carneiros. Boletim da Indústria Animal, v.26, p.275-84, 1969.

17 - MOLINA, E.; BOZA, J.; AGUILERA, J.F. Nutritive value for ruminants of sugar cane bagasse ensiled after spray treatment with different levels of $\mathrm{NaOH}$. Animal Feed Science Technology, v.9. n.1, p.1-17, 1983.

18 - MORGULLIS, S.C.F. Degradabilidade ruminal e digestibilidade aparente do bagaço de cana-de-açúcar tratado por soluçōes alcalinas de cinzas de madeira e hid róxido de sódio. São Paulo, 1992.74 p. Dissertação (Mestrado). Faculdade de Medicina Veterinária e Zootecnia, Universidade de São Paulo.

19 - NOLTE, M.E.; CLINE, J.H.: DEHORITY. B.A.: LOERCH, S.C.; PARKER, C.F. Treatment of wheat straw with alkaline solutions prepared from wood ashes to improve fiber utilization by ruminants. Journal of Animal Science, v, 64, n.3, p.669-77, 1987.

20)- NRC. National Research Council. Nutrient requeriments of sheep. Washinglon, National Academy Press, $1985.99 \mathrm{p}$.

2I - NRC. National Research Council. Nutrient requeriments of dairy cattle. Washington, National Academy Press, $1988.157 \mathrm{p}$

22- PATURAU. J.M. By - Products of the sugar cane industry. Amsterdam. Iilsevier, 1981. p.305-34.

23 - RAMIREZ, R.G.; GARZA, J.; MARTINEZ, J.; AYAI.A, N. Wood ash, sodium hydroxide and urine to increase sorghum straw utilization by sheep. Small Ruminant Research. v.5, p.83-92, 1991

24 - REXEN, F.; THOMSEN, K.V. The effect on digestibility of a new technique for alkali treatment of straw. Animal Feed Science and Technololy, v. I, n. I, p. 73 83, 1976 .

25 - TEH, T.H.; HEMKEN, R.W.; BREMEL „, D.H.; HARMON, R.J. Comparison of buffers on rumen functions, lurnover rate and gastric secretion in Holstein steers. Animal Feed Science and Technololy, v. 17, n. 4, p. 257-70, 1987 